\title{
Improving Recognition of Children Affected by Prenatal Alcohol Exposure: Detection of Exposure in Pediatric Care
}

\author{
Ami C. Bax ${ }^{1}$ - Carrie D. Geurts ${ }^{1}$ - Tatiana N. Balachova ${ }^{1}$
}

Published online: 27 June 2015

(C) Springer International Publishing Switzerland 2015

\begin{abstract}
Early identification of fetal alcohol spectrum disorders (FASDs) is important for providing services and preventing secondary disabilities. Recent studies indicate that many FASDs are undiagnosed, partly because there is a need to improve detection of prenatal alcohol exposure (PAE). The aims of this review are to characterize existing practices for assessing PAE in pediatric care, identify the most efficient, promising methods of detecting PAE, and recognize the knowledge and practice gaps. This review indicates that maternal self-reports remain the most common method utilized in routine clinical practice and highlights promising methods of PAE identification, including a single binge drinking question. The review yields few studies describing existing strategies to assess PAE in pediatric practice and identifies knowledge gaps that need to be addressed for improving recognition of FASDs in pediatric practice.
\end{abstract}

Keywords Prenatal alcohol exposure $\cdot$ Fetal alcohol spectrum disorders $\cdot$ Fetal alcohol syndrome $\cdot$ Neurobehavioral disorder associated with prenatal alcohol exposure (ND-PAE) . Women $\cdot$ Alcohol use $\cdot$ Pregnancy

This article is part of the Topical Collection on Fetal Alcohol Syndrome

Ami C. Bax

ami-bax@ouhsc.edu

Carrie D. Geurts

carrie-geurts@ouhsc.edu

Tatiana N. Balachova

tatiana-balachova@ouhsc.edu

1 Section on Developmental and Behavioral Pediatrics, Department of Pediatrics, University of Oklahoma Health Sciences Center, 1100 N.E. 13th Street, Oklahoma City, OK 73117, USA

\section{Introduction}

Accurate identification of fetal alcohol spectrum disorders (FASDs) is important for providing services and preventing disorders secondary to FASDs. Research indicates that early diagnosis of a child affected by prenatal alcohol exposure (PAE) is associated with better health and life outcomes [1]. However, FASDs remain largely underdiagnosed. The Centers for Disease Control and Prevention (CDC) acknowledged that FASD surveillance is inadequate and that the number of children adversely affected by in-utero exposure to alcohol is underestimated $[2 \cdot \bullet]$. The CDC Fetal Alcohol Syndrome (FAS) Surveillance Network (FASSNet) II [2••] utilized medical records in several states and identified 0.3 children with FAS per 1000 children aged 7-9 years. This figure is significantly lower than the prevalence reported by active case ascertainment studies, in which an expert evaluates children in person; these studies estimated 2-to-7 children affected by FAS and 20-to-50 affected by FASDs per 1000 children in the USA and Western Europe, approximately 2 to $5 \%$ of the population [3••]. Another study reported the prevalence of FASDs among first grade students in a representative Midwestern US community as 6-9 per 1000 children, Partial Fetal Alcohol Syndrome (PFAS) as 11-17 per 1000 children, and the overall rate of FASDs as 24-48 per 1000 children, or 2.4 to $4.8 \%$ [4]. A recent study reported that $86.5 \%$ of foster and adopted youth with FASDs were undiagnosed $(80.1 \%)$ or were diagnosed incorrectly within the FASD spectrum $(6.4 \%)$ prior to a comprehensive evaluation in a specialized clinic $[5 \bullet \bullet$.

Data about women's alcohol consumption indicate that more children may be affected by PAE and be at high risk for FASDs. Per the CDC Behavioral Risk Factor Surveillance System (BRFSS), $7.6 \%$ of pregnant women report consuming alcohol in the past 30 days; $1.4 \%$ report binge drinking, 
defined as consuming four or more alcoholic drinks (standardsized drink equivalents are included in Fig. 1) per occasion during the past 30 days $\left[6^{\bullet}\right]$. According to BRFSS data, among non-pregnant women of childbearing age, the prevalence of any alcohol use in the last 30 days is $51.5 \%$ and the prevalence of binge drinking is $15 \%$, which is higher among young women who are more likely to have children, including women aged 18-24 years $(24.2 \%)$ and $25-34$ years (19.9\%) [6•]. Moreover, about half of pregnancies in the USA are unintended, and a woman may not know she is pregnant until 4-6 weeks of gestation [7]. Given that women often continue to consume alcohol at pre-pregnancy levels until they become aware of their pregnancy [8], there is a high likelihood of PAE before pregnancy recognition, with binge drinking of particular concern because it causes high blood alcohol concentration. Therefore, many pregnancies may be affected by PAE early on, placing children at risk for FASDs.

\section{Drinking and \\ Reproductive Health}

STANDARD-SIZED DRINK EOUIVALENTS APPROXIMATE NUMBER OF STANDARD-SIZED DRINKS IN:
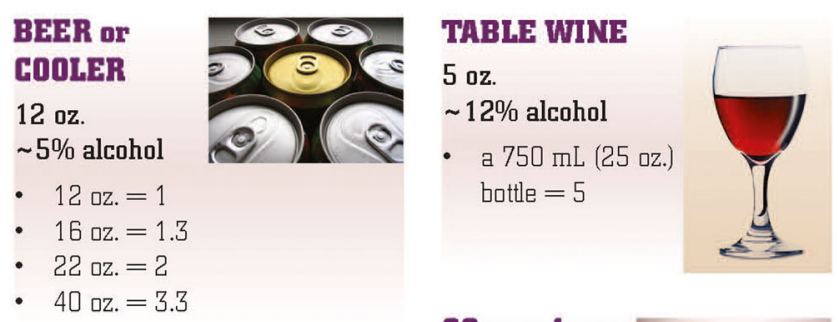

\section{MALT LIOUOR}

8-9 oz.

$\sim 7 \%$ alcohol

- $12 \mathrm{cz} .=1.5$

- $16 \mathrm{cz} .=2$

- 22 oz. $=2.5$

- 40 oz. $=4.5$
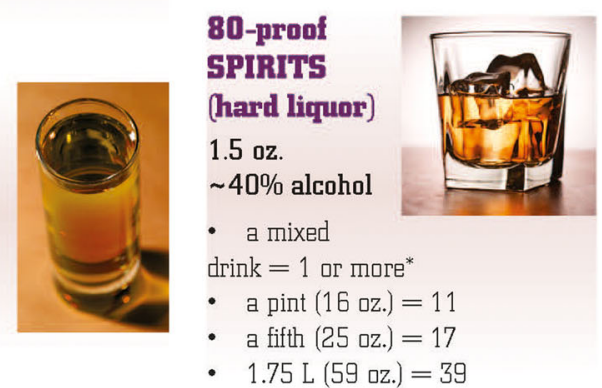

Risky Drinking: More than 7 standard-sized drinks per week or more than 3 per occasion, and drinking when pregnant or trying to become pregnant.

*Note: Depending on factors such as the type of spirits and the recipe, one mixed drink can contain from one to three or more standard drinks.

For urgent assistance with a drinking problem, contact the Substance Abuse \& Mental Health Services Administration at 1-800-273-8255.

Source: National Institute on Alcohol Abuse and Alcoholism. A Pocket Guide for Alcohol Screening and Brief Intervention. 2005. Rockville, MD: National Institutes of Health;2005. Available at: http://pubs.niaaa.nih.gov/publications/Practitioner/PocketGuide/pocket_guide.htm.

Produced by the American College of Gynecologists and Obstetricians (ACOG)

Fig. 1 Standard-sized drink equivalents. (Produced by the American College of Gynecologists and Obstetricians [ACOG]. With Permission from ACOG). [67]
Some cases might not be diagnosed because of the syndromic nature of FASDs, the lack of pathognomonic features, and the negative perceptions associated with alcohol use during pregnancy. The physical features of FAS are often subtle, and the hallmark facial features (smooth philtrum, thin vermillion, small palpebral fissures) are not always fully apparent at birth [9], are difficult to accurately assess without precise measurement [10], and sometimes become less apparent with age. Fox et al. [2••] noted that health care providers inadequately recognize facial features of FAS and often fail to document the features in the patient's records with over half of health providers in this study reporting that they received no training in recognizing FASDs. Several genetic syndromes may present with similar features and require differential diagnosis. Furthermore, physical features may not be present in Partial FAS (PFAS) and in non-dysmorphic FASDs, including Neurobehavioral Disorder Associated with Prenatal Alcohol Exposure (ND-PAE), a new diagnostic category introduced in the Diagnostic and Statistical Manual of Mental Disorders 5th Edition (DSM 5) [11]. The NDPAE was established to recognize individuals who do not meet full criteria for FAS but have impairments in neurocognitive, self-regulatory, and adaptive functioning, and known PAE [11]. The introduction of ND-PAE in the DSM 5 emphasizes the importance of identifying PAE. Unlike FAS, which can be diagnosed without information about PAE history, diagnosing ND-PAE and other non-dysmorphic FASDs requires a confirmed history of more than minimal prenatal exposure. The "minimal" exposure is defined as 1-to-13 drinks/month and no more than 2 drinks per one occasion [11]. Therefore, accurate detection of PAE is necessary to improve identification of children with FASDs and provide services to prevent adverse outcomes.

This paper focuses on detection of PAE to improve the identification of FASDs in pediatric practice. The primary aims of this article are to (1) characterize existing practice and strategies to assess PAE in pediatric care, (2) identify the most efficient, promising methods of detecting PAE, and (3) recognize the knowledge and practice gaps that must be addressed in order to improve recognition of FASDs in pediatric practice.

\section{Review of PAE Screening Practices and Strategies Among Pediatric and Other Clinicians}

A systematic literature search was conducted for studies reporting on PAE screening practices among pediatric clinicians, related guidelines, and barriers to alcohol screening and FASD identification. Because an initial search identified few studies focusing on screening among pediatric clinicians, the search was expanded to include obstetricians, family 
physicians, and other health care providers who provided salient information about PAE screening processes, knowledge base, or competence. Since this review focused on PAE screening among pediatric clinicians, data about other physicians' practices were only included for comparison purposes. No comprehensive review of alcohol screening by other health care providers was conducted. Studies were excluded if they focused on an overview of FASDs/FAS or its diagnostic process, or if they did not address eliciting a maternal selfreport of PAE.

We searched the Ovid Medline and PubMed search engines using a date range of $<1946$ to February Week $22015>$ for "alcohol and pregnancy and screen" AND "physician's practice patterns/or pediatrics/or pediatrician." Adding "selfreport" to this search or substituting it for "screen" yielded the same or fewer results. We also performed a search to find articles on the topic of "Physician awareness and screening for fetal alcohol syndrome" and conducted a general GoogleScholar search on these terms. Our overall search yielded 27 articles. After excluding articles based on the above criteria and those unavailable in full text, two articles were identified as relevant to our primary question of current pediatric screening practices and strategies to identify alcohol consumption during pregnancy. Six additional articles addressed our question in other provider populations and/or assessed pediatric attitudes regarding screening for PAE. An additional search was conducted to examine reports about promising methods of identification of alcohol consumption during pregnancy and barriers and challenges to PAE identification.

\section{Professional Guidelines on Detecting PAE}

The U.S. Surgeon General (2005) issued a statement that health professionals should inquire routinely about alcohol consumption by women of childbearing age, inform them of the risks of alcohol consumption during pregnancy, and advise them not to drink alcoholic beverages during pregnancy [12]. Per these guidelines, pregnant women and women who consider becoming pregnant or may become pregnant should abstain from alcohol. Because approximately half of pregnancies are unplanned and women often do not recognize pregnancy until 4-6 weeks gestation, sexually active women of childbearing age who do not consistently use effective contraception and combine the possibility of pregnancy with risky drinking are at high risk for an alcohol-exposed pregnancy. Although the specific amount of alcohol that can cause an FASD is unknown, the at-risk drinking threshold has been established as $>7$ standard drinks per week or $>3$ standard drinks on a day [13] or $\geq 4$ standard drinks on a single occasion (i.e., binge drinking that results in a $0.08 \%$ gram or higher blood alcohol concentration) [14].

The Substance Abuse and Mental Health Services Administration recommends Screening, Brief Intervention, and
Referral to Treatment (SBIRT) as part of routine health care in numerous settings, including primary care, emergency departments, trauma centers, and other community care centers [15]. The American Academy of Obstetricians and Gynecologists (ACOG) guidelines recommend screening for problematic drinking for all women seeking obstetric-gynecologic care annually and within the first trimester of pregnancy, with early intervention strategies encouraging healthy behaviors and appropriate referrals to a substance abuse professional when indicated $[16,17]$. ACOG recommends the T-ACE (Tolerance, Annoyance, Cut Down, Eye-Opener) and CAGE (Cut Back, Annoyed, Guilty, Eye-Opener) screening tools [17]. The American Academy of Family Physicians recommends universal alcohol use screening for all women of childbearing age and for mothers of patients with clinical features worrisome for FASDs (facial dysmorphia, growth deficiencies, central nervous system impairment) $[18,19]$. The FASD Advisory Workgroup in Canada recognizes that there is no general consensus for screening for alcohol use in women of childbearing age and issues recommendations to improve screening and recording processes of alcohol use among women of childbearing age [20]. The recommendations include a three-level PAE identification model: (1) practicebased approaches, such as motivational interviewing and supportive dialog, implemented by health care providers when talking to women about alcohol use, (2) structured questionnaires, such as CAGE or T-ACE, and (3) biological tests to confirm the presence of alcohol or a drug, the level of exposure, and the presence of multiple drugs.

As part of routine adolescent care, the American Academy of Pediatrics Bright Futures [21-23] recommends a universal substance use screening, brief intervention, and referral to treatment protocol, using CRAFFT, a developmentally appropriate screening tool for alcohol and drug use [21, 22]. However, the framework for screening for parental substance use, including maternal alcohol use during pregnancy in pediatric practice, is limited. The parent is not the pediatrician's patient, so there is no financial reimbursement for parental screening or a brief intervention. Furthermore, if a clinician encounters concerns for problematic substance use, referring the parent to appropriate treatment resources becomes a challenge. Even in the setting of adolescent pregnancy, where both the mother and the child are pediatric patients, there are no clear recommendations for PAE screening, prevention, and treatment with appropriate resources.

A review of the professional guidelines indicates some similarities; however, there is a lack of consistency in methods and recording results in medical records, and no consensus regarding whether all pediatric patients should be screened for PAE $[18,19]$. In order to identify FASDs in children and provide effective treatment, pediatric clinicians ultimately bear the responsibility for detecting PAE and, therefore, are tasked with the challenge of screening the mothers of their 
patients. Although primary care and obstetric clinicians have been tasked with screening and brief interventions to reduce at-risk drinking and prevent FASDs, the identification of maternal alcohol consumption is a relatively new task for pediatric clinicians. There is uncertainty about the actions that a pediatrician is expected to implement if a mother reports alcohol or drug use and about whether the pediatrician is expected to conduct a brief intervention or refer the parent for treatment if indicated. Understanding the current practices of pediatric clinicians is crucial to facing these challenges and improving identification of PAE.

\section{Prenatal Alcohol Screening Practices Among Pediatric and Other Clinicians}

Studies indicated that many pediatricians do not routinely screen their patients for PAE. Vagnerelli et al. [24] reported that $59 \%$ of Italian neonatologists, $78.4 \%$ of Italian pediatricians, and $60 \%$ of Spanish pediatricians endorsed always asking new mothers about ethanol consumption during pregnancy. Over $90 \%$ of all clinicians who reported PAE screening among new mothers used general questions, with less than $10 \%$ reporting use of standardized screening tools [24]. Nanson et al. [25] obtained information about screening practices among Canadian pediatricians and family and general physicians ( $\mathrm{FP} / \mathrm{GP}$ ); pediatricians were less likely to screen mothers about their alcohol use during pregnancy than were FP/GP (58.3 vs $77.4 \%$ ). An earlier survey asked a small sample of Texas practitioners what percentage of colleagues they believed screened for FAS, and the mean response was $30 \%$; however, this study did not specifically examine the practitioners' screening practices [26].

Other medical providers, including obstetricians and $\mathrm{FP} /$ GP, often routinely conduct PAE screening with pregnant and non-pregnant women of childbearing age, but utilization of standard screening tools is limited [26]. A survey of Canadian family physicians revealed that while $75 \%$ reported asking pregnant patients about alcohol use, they were unaware of current screening methods to accurately gauge alcohol use among women [27]. Another Canadian study found that $94 \%$ of family physicians, midwives, and obstetricians reported asking their pregnant patients about alcohol use; however, only $74 \%$ of family physicians, $45 \%$ of obstetricians, and $20 \%$ of midwives utilized a standard screening tool [28]. According to Tough [28], less than $50 \%$ of Canadian health care providers caring for women in the preconception period reported frequently discussing smoking, alcohol use, or addiction history. Arnold et al. [29] found that only $38 \%$ of medical students and residents at a U.S. university reported always screening their pregnant patients for alcohol use, suggesting that there is a need to educate practicing clinicians and trainees about the importance of PAE screening. In general, few articles reported the specific methods used to screen for PAE.

\section{Barriers to Screening for PAE}

Pediatric clinicians face many barriers to accurate detection of PAE, given the social stigma, legal implications, and other factors. Women under-report alcohol consumption because of embarrassment, fear, or beliefs that small amounts of alcohol are inconsequential [30, 31]. Coles [30] noted that alcohol use is reported less frequently than other drug use in medical records. Many adopted and foster care children are at high risk of having had PAE, yet they are often no longer in the care of their biological mother. Clinicians must thus rely upon secondary reports of maternal alcohol use during pregnancy. Because many children have come into the care of other biological relatives or the foster care system, exposure information must often be obtained from medical/social service records or by hearsay. Studies have shown that information yielded in this manner is often less accurate than direct maternal selfreport, contributing to the under-diagnosis of FASDs [30]. When pediatric clinicians have contact with their patients' biological mothers, many find it difficult and feel uncomfortable asking parents about their alcohol use, and if the history is not obtained in a sensitive manner, parents may feel blamed for wrongdoing. Mothers may not reveal their alcohol use during pregnancy because of guilt or fear of legal actions that could result in losing their job, children, or relationships [31].

Clinicians may be reluctant to document PAE due to concerns with mandatory reporting of child maltreatment. ACOG states, "Seeking obstetric-gynecologic care should not expose a woman to criminal or civil penalties or the loss of custody of her children" [16, 17]. Discussing the role of obstetrician-gynecologists in substance abuse reporting and pregnancy, ACOG has expressed concerns that the threat of prosecution is an ineffective strategy in reducing the incidence of alcohol or drug abuse; it may deter women from seeking prenatal care which would be contrary to the child and maternal welfare [16].

The new Child Abuse Prevention and Treatment Act (CAPTA) [32] does not require clinicians to report maternal alcohol use or PAE to Child Protective Services (CPS). However, if a child is diagnosed with FAS/FASDs, clinicians are required to make a referral to CPS so that safe care plans and services are initiated. Additional laws regarding FASDs may differ based on the state in which the clinician practices. These new laws and procedures can improve services for children diagnosed with FASDs but could also increase challenges in obtaining accurate self-reports about alcohol use during pregnancy. When children are older at their first referral, diagnosis relies on the maternal recall of alcohol use after a period of years by a woman who may not remember well or may have some motivation, conscious, or unconscious, to downplay the extent of use [30]. Pediatric clinicians should be aware of the reporting guidelines for reporting FASDs to child welfare and reporting guidelines related to maternal alcohol use within their 
states and include during their regular discussion of consent to evaluation/treatment and disclosure with the family. Health care professionals may be encouraged to work with state legislators to ensure that legislation support education and services to women instead of punishing pregnant women for substance use.

In addition, if a mother reveals alcohol misuse, a pediatrician may feel uncomfortable because of limited skills, time, or resources necessary to adequately respond to the reported problem. Many pediatric clinicians lack screening and brief intervention (SBI) skills and knowledge of community resources on alcohol misuse. Since pediatricians are already tasked with providing SBI for substance abuse in adolescent patients with high risk for pregnancy [21-23, 33], they may be well-poised to assist in providing SBI to mothers of their patients who report at-risk drinking. However, there are barriers to reimbursement for a brief intervention for a parent, a lack of skills to conduct SBI, and no formal pediatric guidelines for such services. Pediatricians need guidelines, training, and resources for improving detection of PAE in their patients and should be provided with specific instructions if they are to help prevent future PAE and FASDs.

Several studies have surveyed clinicians to inquire about their perception of barriers to eliciting a self-report of PAE. A survey of U.S. obstetricians identified several barriers, including physician bias due to own alcohol misuse, lack of training, poor awareness of the problem and its effects, denial that FAS occurs in private practice, time limitations, lack of interest, fear of offending the patient, and fear that patients will lie [34]. Another study reported that family physicians and obstetricians were $60 \%$ more likely to identify time constraints as a barrier to discussing alcohol use during pregnancy with women than were midwives, and almost half of all providers felt that existing information available for discussing PAE not in a useful form for clients [28]. An in-depth focus group of prenatal care providers in San Francisco indicated that practice setting strongly influenced providers' screening and counseling of pregnant women about tobacco, alcohol, and illicit drug use. Providers with larger settings, like county hospitals and the Health Maintenance Organization (HMO), reported more resources, compared with private practitioners. HMO providers were also more likely to report using standardized questionnaires and toxicology screening in pregnancy. In contrast, private practices appeared to offer greater continuity of care and patient-provider relationships [35]. Clinicians have also reported the lack of training and confidence about FAS/FASDs as barriers to identification and diagnosis. In a study of Toronto family physicians, $49 \%$ reported little confidence in their ability to diagnose FAS; $81 \%$ felt their training was inadequate [27]. Italian and Spanish neonatologists and pediatricians also had low confidence in their ability to diagnose FAS and FASDs, with over $50 \%$ feeling that they needed more information regarding FAS/ FASD identification in newborns and children [24].

\section{Biomarkers in Detecting PAE}

Biological tests utilize maternal or child specimens, such as urine, blood, hair, meconium, and umbilical cord samples [36]. Biomarkers of PAE include direct ethanol metabolites/ conjugation products, such as fatty acid ethyl esters (FAEEs), phosphatidylethanol (PEth), ethyl glucuronide (EtG), and ethyl sulfate (EtS), and indirect ethanol-induced protein alterations, such as gamma glutamyltranspeptidase (GGT) [37, 38•, 39-41]. Several studies indicate that biomarkers reveal higher prevalence of alcohol use during pregnancy than do maternal self-reports. A study indicated a fivefold increase in detection of PAE from a meconium FAEE test compared with traditional self-reports in a population-based sample in Canada [42]. A recent review reported PAE prevalence 4.3 times higher when testing meconium than relying on maternal self-reports [43]. These approaches are promising for detecting PAE. However, there are several drawbacks: (1) the methods are costly and may be invasive, (2) nearly twothirds of problem drinkers do not have elevated test values, (3) scores may be elevated due to other factors, such as maternal diabetes or diet, and (4) the methods do not indicate either the amount or the time period of drinking [41]. Traditionally utilized biomarkers, such as urine and blood testing, only provide information about drinking within the last several hours. Other biomarkers, such as meconium or hair testing, provide information about PAE during the last two trimesters of pregnancy. However, these methods are expensive, detect heavier alcohol use, and may be less reliable in detecting moderate or social drinking. In addition, no currently known biomarker can detect exposure during the first trimester.

When a pediatric clinician orders biomarkers on an infant, results may reveal information about the mother, who is not their patient, which raises an ethical concern. Universal biomarker screening raises additional ethical questions. Implementing informed consent to patients about testing and providing biomarker screening only to those who consented reduces the benefits of testing, since patients who consumed alcohol are more likely to refuse testing [44]. However, testing without patient consent would violate the patient's right for autonomy. An additional consideration is that if biomarker screenings were to be routinely used, intervention resources would need to be available [36]. Identification of PAE in older children is even more challenging, as no current laboratory test can detect and quantify PAE in older children.

Some hospitals have implemented targeted biomarker screening and may have established algorithms to determine risk for PAE (e.g., specific concerns due to lack of prenatal care, premature birth, low birth weight, or mother's intoxication at admission; personal communication with Raja 
Nandyal, M.D., and Vadim Ivanov, M.D., neonatologists at the University of Oklahoma Children's Hospital, February 2015). However, there is no commonly accepted algorithm or procedure to record biomarker results in maternal medical charts, and transferring the information to the child's health records had been inconsistent in the USA and Canada [20].

Although biomarker screening could offer advantages over maternal self-reports, research is needed to further validate biomarkers and improve PAE detection [45]. Given these limitations, maternal self-report remains the most common method to assess for PAE in clinical practice.

\section{Strategies for Improving Identification of PAE Through Maternal Self-Report}

Self-reports about alcohol consumption have been demonstrated to reliably reflect women's alcohol consumption. Babor et al. [46] indicated that, in clinical trials of research volunteers, self-report measurements are accurate. Biomarker tests and collateral informant reports do not add sufficient data to warrant their routine use. However, most published data regarding the reliability of self-reports were obtained from volunteers who provided anonymous or confidential information in research studies. A physician asking about alcohol use as part of a clinic appointment in which information is not anonymous and is likely to be included in the patient's medical record makes self-reports more likely to be affected by a social desirability bias. A recent study reported that in-depth research interviews revealed more alcohol use among pregnant women than was recorded during routine clinical care appointments with these women [47].

Several approaches to improve the accuracy of self-reports of alcohol consumption and PAE identification have been identified. Physicians can improve self-report accuracy by asking questions in a sensitive and nonjudgmental manner [33-36] and when embedding questions about alcohol use in the routine prenatal history that includes nutrition, exercise, and avoidance of environmental toxins, such as nicotine/ second-hand smoke. In order to increase accurate and truthful reporting, the woman must be alcohol- and drug-free when interviewed, and the interviewer must use simple language and provide word questions clearly [48]. Asking a woman about her drinking patterns before she became pregnant is another strategy that could elicit more accurate estimates of first-trimester drinking and alcohol use later during pregnancy [49]. Robles et al. [50] found that self-reported alcohol consumption is moderately reliable over 3-month and 5-month time periods, and longer timeframes have been documented to improve sensitivity of self-reports in women of childbearing age [51]. A summary of PAE screening recommendations for pediatric practice is included in Table 1. Figure 2 provides a suggested algorithm for implementing PAE screening and appropriate action plans based upon risks identified within a pediatric practice.

\section{Screening Instruments to Identify Alcohol Use During Pregnancy}

A number of screening measures have been implemented for screening for at-risk drinking in different settings. Two of the instruments have been designed and validated specifically to detect the risk for alcohol use during pregnancies in women, TWEAK (Tolerance, Worried, Eye-opener, Amnesia, Kut down) and T-ACE [52-54]. These measures have been tested in diverse obstetric samples and demonstrated good performance in PAE identification [52, 55-57]. In addition, the AUDIT-C (Alcohol Use Disorders Identification Test alcohol consumption questions) has demonstrated sensitivity in identification of prenatal alcohol use among women [58]. Most women who consume alcohol during pregnancy do not meet criteria for alcohol use disorders and may not be identified with commonly used instruments, such as the standard AUDIT (Alcohol Use Disorders Identification Test) [59]. For example, the CAGE questionnaire and SMAST (Short Michigan Alcoholism Screening Test) were designed to identify hazardous and harmful drinking and alcohol dependency and were tested in specific populations (e.g., heavy drinkers, males) [60]. These instruments do not accurately identify drinking among women $[15,16,58]$. T-ACE and TWEAK, simple screening instruments designed for identifying women who consume alcohol during pregnancy, are particularly valuable and detecting the risk for PAE.

\section{Single Question to Identify Alcohol Use During Pregnancy}

Even brief instruments consisting of a few questions may be difficult to implement in a busy pediatric practice. Recent studies indicate that a single question can be used for screening. The National Institute on Alcohol Abuse and Alcoholism recommended the Single "Binge" Drinking Question (SBDQ) ("How often did you have $\geq 4$ drinks on one occasion?") to screen people whose drinking puts them at risk of an alcohol use disorder [13]. Recent studies found that the SBDQ accurately identified patients with at-risk drinking in primary care [61] and also identified women whose drinking placed them at risk of PAE [62-64]. These findings support the use of SBDQ to identify prenatal alcohol use. However, the studies were conducted in research settings, and additional research is needed to evaluate the performance of the measure in pediatric care settings. It is reasonable, though, to conclude that the SBDQ and screening instruments, including T-ACE and TWEAK, are feasible and effective for PAE identification and can be recommended for implementation in pediatric care settings. 


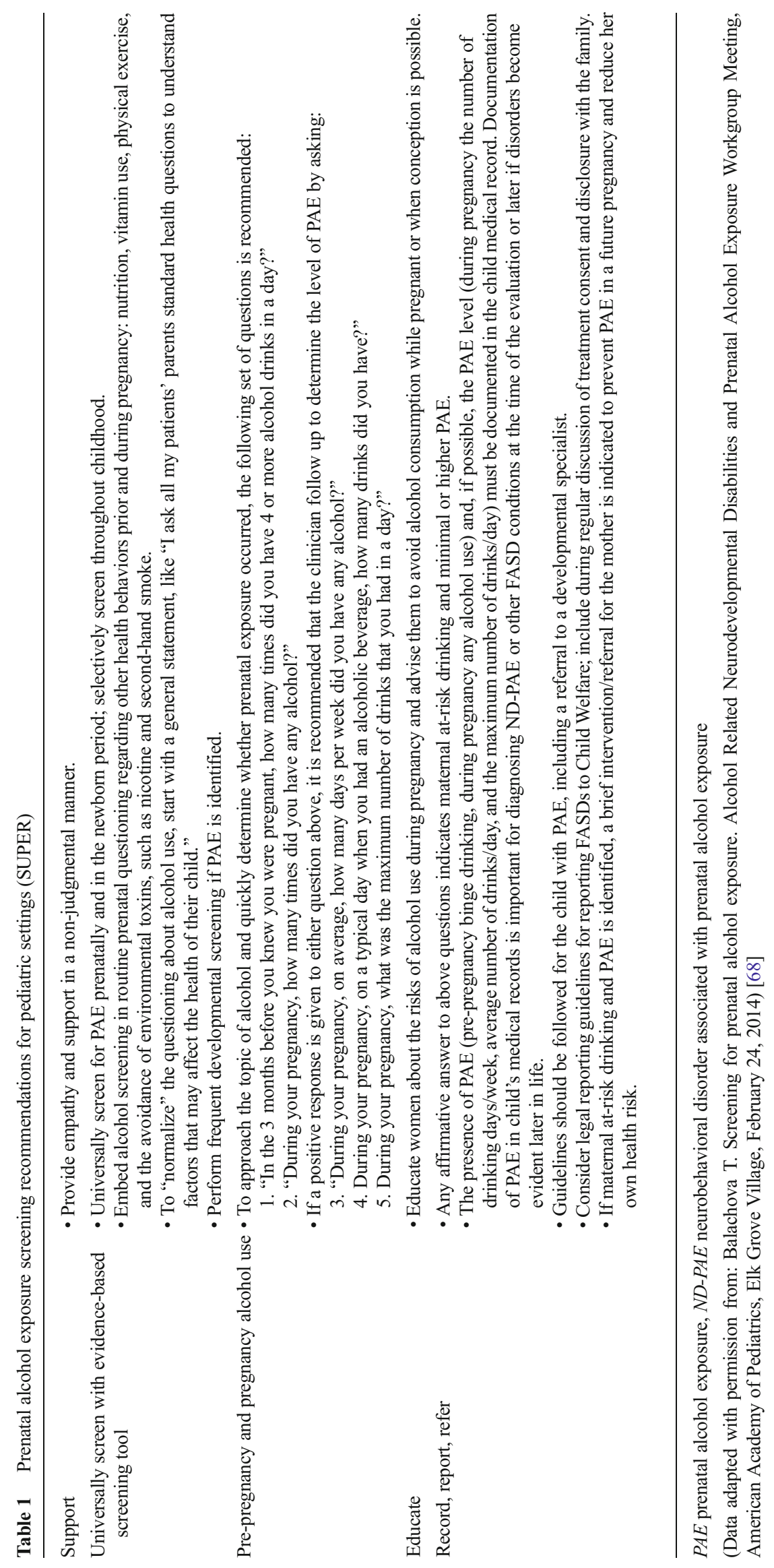


Questions to guide PAE identification/intervention for pediatric patient and mother

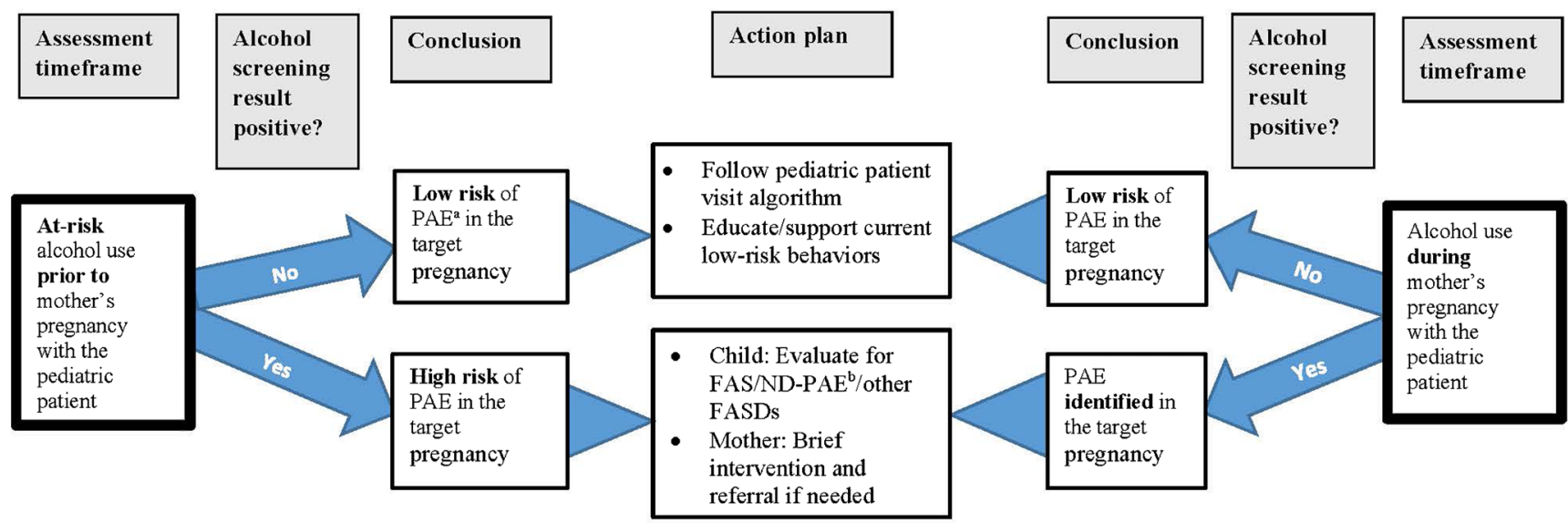

Questions to Guide Primarv Prevention of PAE in Future Pregnancies and FASDs in Pediatric Patients

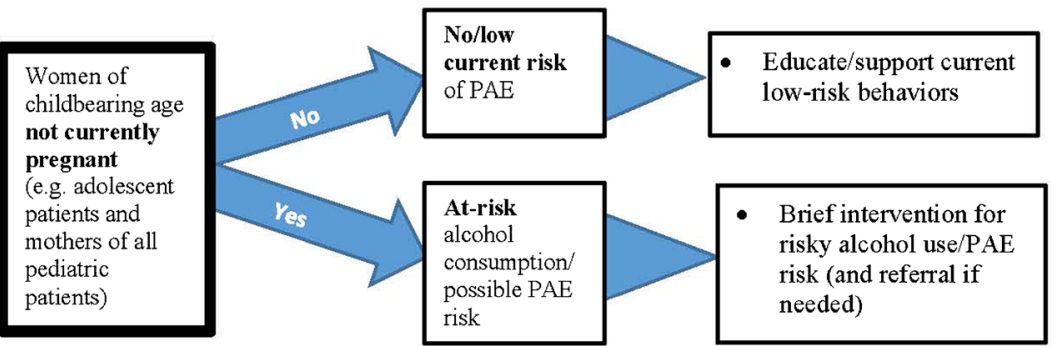

aPAE: prenatal alcohol exposure; bND-PAE: neurobehavioral disorder associated with prenatal alcohol exposure

(Data adapted with permission from: Balachova T. Screening for prenatal alcohol exposure. Alcohol Related Neurodevelopmental Disabilities and Prenatal Alcohol

Exposure Workgroup Meeting, American Academy of Pediatrics, Elk Grove Village, February 24, 2014) [68]

Fig. 2 Suggested algorithms for assessing prenatal alcohol exposure: action plan

\section{Conclusions}

Screening women for at-risk drinking and high risk for alcohol-exposed pregnancies has been recommended for primary care, and is widely accepted as a part of routine care by many practitioners. Recent findings regarding the clinical features and prevalence of FASDs and new DSM 5 guidelines for diagnosing ND-PAE highlight the importance of improving PAE identification in pediatric settings. Thus, pediatric clinicians are tasked with eliciting accurate maternal self-reports of alcohol use during pregnancy. Since women are more likely to provide accurate reports of prenatal alcohol use retrospectively, pediatric clinicians should be reassured that they are in the best position to engage in this discussion and can prompt accurate information about PAE.

Although challenges in PAE identification have been reported, a significant body of research and clinical guidelines support pediatric clinicians in identification of PAE. Currently, maternal self-reports about alcohol use are a more practical method of PAE detection than biological methods. Several self-report methods, including the SBDQ, have shown validity [52, 55, 63-66]. These approaches need further evaluation in clinical settings to determine their accuracy when implemented by pediatric professionals. Studies have indicated that many clinicians feel they lack the confidence, skills, and time to effectively screen for PAE, and that they need training in effective screening methods that require minimal time [24, 27, 34]. However, few studies have examined the general frequency and methods of PAE screening practices used by pediatricians. Additional measures, such as screening by nurses and other health care professionals, incorporating screening tools in electronic medical records, and utilization of tablets/electronic system screening, can be utilized in pediatric offices. By improving recognition of PAE via accurate maternal self-reports, pediatric clinicians can improve early identification of FASDs, ensure services and early interventions for children with FASDs to prevent secondary disabilities, and potentially contribute to prevention of PAE in future pregnancies. 
Acknowledgments The authors wish to thank Kathy Kyler of the University of Oklahoma Health Sciences Center for her help with editing the manuscript. The publication was partially supported by Research Grant Number R01AA016234 from the National Institutes of Health/National Institute on Alcohol Abuse and Alcoholism and Fogarty International Center (Brain Disorders in the Developing World: Research Across the Lifespan) and a Cooperative Agreement with the University of Missouri (Sub-award Number C00399-76; DHHS/CDC Grant Award U84DD000884). The contents of this article are solely the responsibility of the authors and do not necessarily represent the official views of the $\mathrm{NIH}$ and $\mathrm{CDC}$.

\section{Compliance with Ethics Guidelines}

Conflict of Interest Ami C. Bax, Carrie D. Geurts, and Tatiana N. Balachova declare that they have no conflict of interest.

Human and Animal Rights and Informed Consent This article does not contain any studies with human or animal subjects performed by any of the authors.

\section{References}

Papers of particular interest, published recently, have been highlighted as:

- Of importance

•- Of major importance

1. Streissguth AP, Bookstein FL, Barr HM, et al. Risk factors for adverse life outcomes in fetal alcohol syndrome and fetal alcohol effects. Dev Behav Pediatr. 2004;25(4):228-38.

2.• Fox DJ, Pettygrove S, Cunniff C, et al. Fetal alcohol syndrome among children aged 7-9 years-Arizona, Colorado, and New York, 2010. MMWR Morb Mortal Wkly Rep. 2015;64(3):54-7. Although FAS surveillance is challenging, the CDC FAS Surveillance Network strives to provide routine updates on the estimated prevalence of FAS in the USA, which according their studies, has remained at $0.3 / 1000$ children.

3.• May PA, Gossage JP, Kalberg WO, et al. Prevalence and epidemiologic characteristics of FASD from various research methods with an emphasis on recent in-school studies. Dev Disabil Res Rev. 2009;15(3):176-92. doi:10.1002/ddrr.68. Results of this study estimated that approximately 2 to $5 \%$ of children in the USA and Western Europe are affected by FAS and FASDs.

4. May PA, Baete A, Russo J, et al. Prevalence and characteristics of fetal alcohol spectrum disorders. Pediatrics. 2014. doi:10.1542/ peds.2013-3319.

5.• Chasnoff IJ, Wells AM, King L. Misdiagnosis and missed diagnoses in foster and adopted children with prenatal alcohol exposure. Pediatrics. 2015;135(2):264-70. doi:10.1542/peds.2014-2171. This study reports the high rates of FASD under-diagnosis and misdiagnosis in foster and adopted youth.

6. Centers for Disease Control and Prevention. Alcohol Use and Binge Drinking Among Women of Childbearing Age - United States, 2006-2010. 2012;534-8. The CDC Behavioral Risk Factor Surveillance System summarized the prevalence of alcohol use in women of childbearing age, highlighting overall rates of $51.5 \%$ for any alcohol use in the last 30 days and $15 \%$ for binge drinking.

7. Finer L, Zolna M. Unintended pregnancy in the United States: Incidence and disparities, 2006. Contraception. 2011;84(5):478785 .
8. Tough S, Tofflemire K, Clarke M, et al. Do women change their drinking behaviors while trying to conceive? An opportunity for preconception counseling. Clin Med Res. 2006;4:97-105.

9. Stoler JM, Holmes LB. Under-recognition of prenatal alcohol effects in infants of known alcohol abusing women. J Pediatr. 1999;135(4):430-6.

10. Astley S. Validation of the fetal alcohol spectrum disorder (FASD) 4-digit diagnostic code. J Popul Ther Clin Pharmacol. 2013;20(3): 416-67.

11. Diagnostic and statistical manual of mental disorders. 5th ed. Washington DC: American Psychiatric Association; 2013.

12. US Surgeon General. US Surgeon General Releases Advisory on Alcohol Use in Pregnancy. February 21, 2005. http://come-over.to/ FAS/SurGenAdvisory.htm. Accessed April 292015.

13. National Institute on Alcohol Abuse and Alcoholism. Helping patients who drink too much: A clinician's guide In: Department of Health and Human Services, editor. February 21, 2005.

14. National Institute on Alcohol Abuse and Alcoholism. NIAAA Newsleter Winter 2004. In: Department of Health and Human Services, editor. 2004. p. 3.

15. National Institute on Alcohol Abuse and Alcoholism. Alcohol screening and brief intervention for youth: A practitioner's guide. In: Administration Substance Abuse and Mental Health Services Administration, editor. 2011. p. 6.

16. American College of Obstetricians and Gynecologists. Committee opinion no. 473: substance abuse reporting and pregnancy: the role of the obstetrician-gynecologist. Obstet Gynecol. 2011;117(1):200 1.

17. American College of Obstetricians and Gynecologists. Committee opinion no. 496: At-risk drinking and alcohol dependence: obstetric and gynecologic implications. Obstet Gynecol. 2011;118(2, Part 1): 383-8. doi:10.1097/AOG.0b013e31822c9906.

18. Wattendorf DJ, Muenke M. Fetal alcohol spectrum disorder. Am Fam Physician. 2005;72(2):279-85.

19. Morantz C. CDC releases guidelines on identifying and referring persons with fetal alcohol syndrome: practice guidelines. Am Fam Physician. 2006;1(73):916-22.

20. Sarkar M, Burnett M, Carrier S, et al. Screening and Recording of alcohol use among women of child-bearing age and pregnant women. Can J Clin Pharmacol. 2009;16(1):e242-63.

21. Hagan J, Shaw J, Duncan P, editors. Bright futures: guidelines for health supervision of infants, children, and adolescents. 3rd ed. Elk Grove Village: American Academy of Pediatrics; 2008.

22. American Academy of Pediatrics. Substance use screening, brief interventions, and referral to treatment for pediatricians. Pediatrics. 2011;128:e1330-40.

23. Performing preventative services: a bright futures handbook. American Academy of Pediatrics. Tanski S, Garfunkel LC, Duncan PM, Weitzman M, editors. 2010.

24. Vagnarelli F, Palmi I, Garcia-Algar O, et al. A survey of Italian and Spanish neonatologists and paediatricians regarding awareness of the diagnosis of FAS and FASD and maternal ethanol use during pregnancy. Biomed Cent Pediatr. 2011;11:51.

25. Nanson JL, Bolaria R, Snyder RE, et al. Physician awareness of fetal alcohol syndrome: a survey of pediatricians and general practitioners. Can Med Assoc J. 1995;152(7):1071-6.

26. Conrad C. Physician awareness and screening for fetal alcohol syndrome. J Health Hum Serv Adm. 2000;22(3):257-76.

27. Nevin AC, Christopher P, Nulman I, et al. A survey of physicians knowledge regarding awareness of maternal alcohol use and the diagnosis of FAS. Biomed Cent Fam Pract. 2002;3:2.

28. Tough SC, Clarke M, Hicks M, et al. Attitudes and approaches of Canadian providers to preconception counselling and the prevention of fetal alcohol spectrum disorders. Int J Fundam Appl Sci. 2005;3, e3. 
29. Arnold K, Burke M, Decker A, et al. Fetal alcohol spectrum disorders: knowledge and screening practices of university hospital medical students and residents. J Popul Ther Clin Pharmacol. 2013;20(1):e18-25.

30. Coles CD. Discriminating the effects of prenatal alcohol exposure from other behavioral and learning disorders. Alcohol ResHealth. 2011;34(1):42-50.

31. Barr HM, Streissguth AP. Identifying maternal self-reported alcohol use associated with fetal alcohol spectrum disorders. Alcohol Clin Exp Res J. 2001;25(2):283-7.

32. The Child Abuse Prevention and Treatment Act Reauthorization Act of 2010. In: U.S. Department of Health and Human Services Administration for Children and Families Administration on Children, Youth and Families Children's Bureau, editors. 2010.

33. American Academy of Pediatrics. Contraception for adolescents. Pediatrics. 2014;134(4):e1244-56.

34. Donovan CL. Factors predisposing, enabling and reinforcing routine screening of patients for preventing fetal alcohol syndrome: a survey of New Jersey physicians. J Drug Educ. 1991;21(1):35-42.

35. Gilbert P, Herzig K, Thakar D, et al. How health care setting affects prenatal providers' risk reduction practices: a qualitative comparison of settings. Women Health. 2007;45(2):41-57. doi:10.1300/ J013v45n02_03

36. Gifford AE, Farkas KJ, Jackson LW, et al. Assessment of benefits of a universal screen for maternal alcohol use during pregnancy. Birth Defects Res A Clin Mol Teratol. 2010;88(10):838-46. doi:10.1002/ bdra.20731.

37. Bearer C, Santiago L, O'Riordan M, et al. Fatty acid ethyl esters: quantitative biomarkers for maternal alcohol consumption. J Pediatr. 2005;146(6):824-30.

38. Joya X, Friguls B, Ortigosa S, et al. Determination of maternal-fetal biomarkers of prenatal exposure to ethanol: a review. J Pharm Biodmed Anal. 2012;69:209-12. This is an important review of maternal-fetal biomarkers of prenatal exposure to ethanol.

39. Lendoiro E, Concheiro M, González-Colmenero E, et al. Maternal hair analysis for the detection of illicit drugs, medicines and alcohol exposure during pregnancy. J Popul Ther Clin Pharmacology. 2012;19:e303.

40. Wurst F, Kelso E, Weinmann W, et al. Measurement of direct ethanol metabolites suggests higher rate of alcohol use among pregnant women than found with the AUDIT - a pilot study in a population-based sample of Swedish women. Am J Obstet Gynecol. 2008;198(4):407.e1-.e5.

41. Bakhireva L, Savage D. Biomarkers of fetal alcohol exposure and fetal alcohol effects. Alcohol Res Health. 2011;34:56-63.

42. Gareri J, Lynn H, Handley M, et al. Prevalence of fetal ethanol exposure in a regional population-based sample by meconium analysis of fatty acid ethyl esters. Ther Drug Monit. 2008;30(2):239-45.

43. Lange S, Shield K, Koren G, et al. A comparison of the prevalence of prenatal alcohol exposure obtained via maternal self-reports versus meconium testing: a systematic literature review and meta-analysis. BMC Pregnancy Childbirth. 2014;14(1):127. doi:10.1186/ 1471-2393-14-127.

44. Zelner I, Shor S, Lynn H. Neonatal screening for prenatal alcohol exposure: assessment of voluntary maternal participation in an open meconium screening program. Alcohol. 2012;46:269-76.

45. Littner Y, Bearer CF. Review detection of alcohol consumption during pregnancy-current and future biomarkers. Neurosci Biobehav Rev. 2007;31(2):261-9.

46. Babor TF, Steinberg K, Anton R, et al. Talk is cheap: measuring drinking outcomes in clinical trials. J Stud Alcohol Drugs. 2000;61(1):55-63.

47. Hughes SC, Chambers CD, Kassem N, et al. Inconsistent report of pre-pregnancy recognition alcohol use by Latinas. Matern Child Health J. 2009;13(6):857-64. doi:10.1007/s10995-008-0416-3.
48. Brown J, Kranzier HR, Del Boca FK. Self-reports by alcohol and drug abuse inpatients: factors affecting reliability and validity. Br J Addict Alcohol Drugs. 1992;87(7):1013-24.

49. Day NL, Cottreau CM, Richardson GA. Epidemiology of alcohol, marijuana, and cocaine use among women of childbearing age and pregnant women. Clin Obstet Gynecol. 1993;36:237-45.

50. Robles N, Day NL. Recall of alcohol consumption during pregnancy. J Alcohol Drug Educ. 1990;51(5):403-7.

51. Nayak MB, Kaskutas LA. Risky drinking and alcohol use patterns in a national sample of women of childbearing age. Addiction. 2004;99(11):1393-402.

52. Sokol R, Martier S, Ager J. The T-ACE questions: practical prenatal detection of risk-drinking. Am J Obstet Gynecol. 1989;160:86371

53. Russell M, Martier SS, Sokol RJ, et al. Detecting risk drinking during pregnancy: a comparison of four screening questionnaires. Am J Public Health. 1996;86(10):1435-9.

54. Russell M, Martier SS, Sokol RJ, et al. Screening for pregnancy risk-drinking. Alcohol Clin Exp Res J. 1994;18(5):1156-61.

55. Chang G. Screening and brief intervention in prenatal care settings. Alcohol Research and Health. 2004/2005;28(2):80-4.

56. Sokol RJ, Delaney-Black V, Nordstrom B. Fetal alcohol spectrum disorder. J Am Med Assoc. 2003;290(22):2996-9. doi:10.1001/ jama.290.22.2996.

57. Chang G, WIlkins-Haug L, Berman S, et al. Alcohol use and pregnancy: improving identification. Obstet Gynecol. 1998;91(6):892-8.

58. Burns E, Gray R, Smith LA. Brief screening questionnaires to identify problem drinking during pregnancy: a systematic review. Addiction. 2010;105(4):601-14. doi:10.1111/j.1360-0443.2009. 02842.x.

59. Babor TF, Biddle-Higgins JC, Saunders JB, et al. AUDIT: the Alcohol Use Disorders Identification Test: guidelines for use in primary health care. In: Organization WH, editor. Geneva, Switzerland; 2001.

60. Bradley K, Boyd-Wickizer J, Powell SH, et al. Alcohol screening questionnaires in women: a critical review. J Am Med Assoc. 1998;280(2):166-71. doi:10.1001/jama.280.2.166.

61. Smith PC, Schmidt SM, Allensworth-Davies D, et al. Primary care validation of a single-question alcohol screening test. J Gen Intern Med. 2009;24:783-8.

62. Balachova T, Sobell LC, Agrawal S, et al. Using a single binge drinking question to identify Russian women at risk for an alcohol-exposed pregnancy. Addict Behav. 2015;46:53-7. doi:10. 1016/j.addbeh.2015.03.003.

63. Johnson KE, Sobell MB, Sobell LC. Using one question to identify women at risk for an alcohol-exposed pregnancy. J Am Osteopath Assoc. 2010;110(7):381-4.

64. Dum M, Sobell LC, Sobell MB, et al. A quick drinking screen for identifying women at risk for an alcohol-exposed pregnancy. Addict Behav. 2009;34(9):714-6. doi:10.1016/j.addbeh.2009.04. 001.

65. Flynn HA, Marcus SM, Barry KL, et al. Rates and correlates of alcohol use among pregnant women in obstetrics clinics. Alcohol Clin Exp Res. 2003;27(1):81-7. doi:10.1111/j.1530-0277.2003. tb02725.x.

66. Sobell LC, Sobell MB. Alcohol Timeline Followback (TLFB). In: Rush J, First MB, Blacker D, editors. Handbook of psychiatric measures. 2nd ed. Washington: American Psychiatric Association; 2008. p. 466-8.

67. National Institute on Alcohol Abuse and Alcoholism. A pocket guide for alcohol screening and brief intervention. Rockville: National Institutes of Health; 2005.

68. Balachova T. Screening for prenatal alcohol exposure. Alcohol Related Neurodevelopmental Disabilities and Prenatal Alcohol Exposure Workgroup Meeting, American Academy of Pediatrics, Elk Grove Village, February 24, 2014. 